Original scientific paper

\title{
VEHICLE ROUTING PROBLEM WITH SOFT TIME WINDOWS OF CARGO TRANSPORT O2O PLATFORMS
}

\author{
Liu, M. L. ; Zhang, C. "\#; Wu, Q. L. ${ }^{* *} \&$ Meng, B. R.* \\ ${ }^{*}$ School of Transportation and Logistics, East China Jiaotong University, Nanchang, 330013, China \\ ${ }^{* *}$ School of Civil Engineering and Architecture, East China Jiaotong University, Nanchang, 330013, \\ China \\ E-Mail: 0266@ecjtu.edu.cn ( ${ }^{\#}$ Corresponding author)
}

\begin{abstract}
To attain the goal of cost minimization, a vehicle routing model for cargo transport $\mathrm{O} 2 \mathrm{O}$ platforms were established in this study. In consideration of differences in vehicle origin in the traditional vehicle routing problem as well as the one-to-one corresponding relationship between cargo owners' pickup and delivery points, constraint conditions such as half-open, multiple depot, multiple vehicle type, origindestination pair, loading limit, and soft time window constraints were introduced into the proposed model. Given the model characteristics, an improved genetic algorithm, which is commonly used in vehicle routing problem, was used as the solving tool. The nearest matching method currently used by cargo transport $\mathrm{O} 2 \mathrm{O}$ platforms was simulated using the simulation software AnyLogic. Moreover, vehicle-cargo orders on a platform within a certain time period were selected and allocated, and a matching scheme was obtained. Then, the optimized matching scheme for the same order was calculated using the improved genetic algorithm. Results show the comprehensive cost obtained by the improved genetic algorithm is $21.14 \%$ lower than that of obtained by the nearest matching method.

(Received in February 2021, accepted in April 2021. This paper was with the authors 2 weeks for 1 revision.)
\end{abstract}

Key Words: Cargo Transport O2O, Vehicle Routing Problem, Soft Time Window, Improved Genetic Algorithm

\section{INTRODUCTION}

Cargo transport $\mathrm{O} 2 \mathrm{O}$ platforms are a new mode combining online promotion and offline consumption via mobile Internet technology and uniquely advantaged in solving pain spots of traditional highway cargo transport, such as vehicle-cargo information asymmetry and low vehicle load ratio. The core competitiveness of cargo transport $\mathrm{O} 2 \mathrm{O}$ platforms lies in their capability to solve the optimized resource allocation problem of mass cargo transport orders. However, such platform encounters difficulties in solving vehicle-cargo matching and vehicle routing problem (VRP) with efficient and optimized algorithms.

Vehicle-cargo matching and route optimization, which such platforms are dedicated to solving, are applications of a classical problem, namely, the VRP [1], which is discussed in academic circles for real scenarios in the cargo transport industry. In theoretical circles, recent VRP-related studies began with constraint conditions, such as change-of-vehicle capacity, service time windows, and orders' special needs, and derived a VRP with time windows (VRPTW) [2], a split delivery VRP (SDVRP), a multi-depot VRP (MDVRP), a half-open VRP, and so on. However, most studies are partial to improving theoretical small-scale solving algorithms, and how to apply complex large-scale VRP solving algorithms to actual scenarios is an important topic that has yet to be explored. In the present study, cargo transport $\mathrm{O} 2 \mathrm{O}$ platforms, which are an emerging force in solving vehicle-cargo information asymmetry in the field of highway cargo transport, are taken as the study objects, and efforts are made to provide efficient solutions to the vehicle-cargo matching problem and VRP of vehicle and cargo owners. In terms of related research, a single-depot origin-destination (OD) pair VRPTW was examined in University of Bologna [3], and the cargo transport $\mathrm{O} 2 \mathrm{O}$ citywide peddle car-cargo matching problem with time windows was investigated in Huazhong University of Science and 
Technology [4]. Based on the aforementioned research, innovations are made in the current study. The cargo transport $\mathrm{O} 2 \mathrm{O}$ vehicle-cargo matching and VRP examined in this study include constraint conditions such as soft time window, vehicle volume limitation, OD pair (one-to-one origin and destination correspondence), multiple depot, multiple vehicle type, and half-open constraints, thereby catering to actual needs to a certain extent. Moreover, the nearest matching process of cargo transport $\mathrm{O} 2 \mathrm{O}$ platforms is simulated via AnyLogic to verify the feasibility of the improved genetic algorithm (GA) for cargo transport $\mathrm{O} 2 \mathrm{O}$ platforms.

\section{STATE OF THE ART}

In theoretical circles, efforts were made to improve various VRP optimization algorithms and continuously broaden the practical applications of digital and intelligent vehicle-cargo matching platforms.

For the solving of optimization algorithms, Lagos et al. [5] examined a VRPTW with simultaneous pickup and delivery and minimized the total route distance to satisfy clients' delivery and pickup demands simultaneously. The results of the study showed that an improved particle swarm optimization (PSO) algorithm can be used to find a relatively ideal solution. Erdoan [6] developed an open source Excel-based tool to solve the VRP of multiple vehicle type and gave the solver and calculation results of the solving algorithm for benchmark examples from the related literature. Montero et al. [7] discussed a VRP with pickup and delivery (VRPPD) and developed a local search program based on integer linear programming (ILP), and the results of the calculation example verified the potential of the method in solving VRPPD. Tiwari et al. [8] proved a modified environmental adaptation method can evade the incidence of being struck into local minima. Alaia et al. [9] investigated a multi-vehicle, multidepot pickup and delivery problem with time windows and solved a benchmark example using a GA and PSO algorithm, and the results showed that the GA algorithm is better than the PSO algorithm. Jiang [10] used a dynamic clustering algorithm to solve the model that considered the order similarity and the picking time. Bulhões et al. [11] established a VRP with a servicelevel constraint and solved a standard calculation example with a mixed algorithm with population management, and the results indicated that the mixed GA with population management can provide a high-quality solution by balancing the service level and cost. Gallegos-Acosta et al. [12] developed a manipulation of metaheuristics to decrease computational requirements. The results of the above studies showed that various routing problems can be solved through the use of different types of heuristic algorithms, such as an adaptive large neighbourhood search algorithm, a PSO algorithm, ILP, a GA, and a quantum ant colony algorithm. The superiority of these algorithms when applied to benchmark or smallscale calculation examples was examined, but their performance in solving actual large-scale problems has yet to be verified.

In terms of practical applications, Campelo et al. [13] developed a set of algorithm routines reduced according to instance size for clients with dynamic daily delivery and service demands, selected a pharmaceutical distribution company as an example, and planned distribution routes for multiple warehouses and 100 orders. Meanwhile, Sevkli and Güler [14] constructed an OVRP to develop an optimization plan for a media company in Turkey to deliver newspapers to different sites, solved the problem using a variable neighbourhood search-based algorithm, and improved the efficiency of the distribution scheme. Hannan et al. [15] proposed an improved PSO algorithm to solve a garbage collection route optimization plan, which served as a valuable garbage collection and route optimization tool. Poonthalir and Nadarajan [16] presented and solved a fuel-efficient green VRP using a PSO algorithm with a time-varying acceleration coefficient and greedy mutation operator, and the results showed that a route plan involving minimum fuel consumption can be obtained under variable speed. Moreover, hyper- 
heuristic algorithms were used to solve practical VRP applications, but most are restricted to traditional origin-to-destination routing, thereby solving a two-variable routing problem.

Emerging cargo transport $\mathrm{O} 2 \mathrm{O}$ platforms have integrated mass truck resources and cargo owner order resources, and the reasonable distribution of resources from the global optimum angle is a new type of VRP representation, which is a classical problem, in the era of mobile Internet technology. The operation is a three-variable routing problem is the challenge of solving this problem with hyper-heuristic algorithms lies in the fact that cargo transport $\mathrm{O} 2 \mathrm{O}$ platforms. In addition, a cargo owner's pickup point and delivery point must have a one-to-one corresponding relation, which can aggravate the difficulty of solving this problem. Presently, few studies exist on this topic; a VRP model meeting actual needs with constraint conditions is constructed. In addition, an improved GA and simulation software AnyLogic are used to solve the VRP. Through a comparison of results, the feasibility of the improved GA in obtaining an optimal vehicle routing plan in actual platform operation is verified.

The remainder of this study is organized as follows. Section 3 establishes the models for the vehicle-cargo matching scheme and VRP of cargo transport $\mathrm{O} 2 \mathrm{O}$ platforms, expounds on the simulation logic of AnyLogic, and constructs the rules for the improved GA for solving the research problem. Section 4 uses AnyLogic and improved GA to solve the problem of cargo transport $\mathrm{O} 2 \mathrm{O}$ platform orders and compares the results obtained by the two methods in terms of transportation cost, service cost, the penalty cost of the time window deviation, and so on. The final section summarizes the study and provides related conclusions.

\section{METHODOLOGY}

\subsection{Problem description}

This study aims to obtain a reasonable allocation of tanker pool resources and sources of goods in cargo transport $\mathrm{O} 2 \mathrm{O}$ platforms as well as a global optimal solution. Given these objectives, the minimization of the comprehensive cost of cargo and vehicle owners was taken as the objective, and soft time window, vehicle volume limit, OD pair, multiple depot, multiple vehicle type, and half-open constraints were employed as the constraint conditions to construct the VRP model.

(1) For the soft time window constraint, according to the interviews conducted by the authors, the cargo owners had time window requirements after placing orders on a platform. However, in case of any time window deviation, the vehicle owners were allowed to receive orders and deliver goods, and satisfaction may be reduced. Therefore, a penalty cost for time window deviation (soft time window constraint) was added to the objective function.

(2) Only the vehicle volume limit was considered in this study, because according to the investigation conclusions of the authors, the orders undertaken by vehicle owners on cargo transport $\mathrm{O} 2 \mathrm{O}$ platforms generally did not exceed the specified weight, whereas whether the vehicle volume was sufficient was considered in crowd ordering.

(3) Multiple depot, multiple vehicle type, half-open, and OD pair constraints were employed as constraint conditions, because the actual operation scenario of a cargo transport $\mathrm{O} 2 \mathrm{O}$ platform was simulated in this study. First, the orders undertaken by a vehicle owner from a cargo owner involved two business operations, namely, pickup and delivery, and the cargo owner's pickup point and delivery point must demonstrate a one-to-one corresponding relationship, namely, the OD pair constraint condition. Second, the origin of each vehicle was not the pickup point, and the vehicles were located in places with geographical coordinates independent of the pickup point and delivery point, that is, the multiple depot constraint condition. Third, the vehicle type in each depot was not a single type but diversified types. In the end, each vehicle can park at the last delivery point without returning to the initial position, that is, the half-open constraint condition, because the travel route taken by vehicle owners in 
actual situations aims to maximize revenue and receive orders continuously only if suitable orders exist, not taking the origin as the destination.

\subsection{Problem hypotheses}

(1) The platform has adequate time to optimize the vehicle-cargo matching scheme.

(2) Multiple depots are distributed within the region, and only one vehicle is in each depot.

(3) The vehicle type varies from depot to depot. Only the volume limit, and not the loading limit, is considered, without a maximum mileage limit.

(4) Each cargo owner (including the pickup point and delivery point) must and is allowed to be visited only once.

(5) Each cargo owner has time window requirements for pickup and delivery.

(6) Each vehicle can serve multiple sub-routes while meeting clients' time window requirements.

\subsection{Symbol definition}

(1) Parameter definition

$P=\{1,2, \ldots, n\}$ : set of all pickup points of the cargo owner, and $P_{1}, P_{2}, \ldots, P_{n}$ represents $n$ pickup points of the cargo owner.

$D=\{n+1, n+2, \ldots, 2 n\}:$ set of all delivery points of the cargo owner, and $D_{n+1}, D_{n+2}, \ldots, D_{2 n}$ denotes $n$ delivery points, where the pickup point $P_{1}$ corresponds to the delivery point $D_{n+1}$, and by parity of reasoning, pickup point $P_{n}$ corresponds to delivery point $D_{2 n}$.

$K=\{1,2, \ldots, m\}$ : set of all vehicles, and $K_{1}, K_{2}, \ldots, K_{m}$ represents $m$ delivery vehicles.

$A=P \cup D \cup K$ : set of all pickup points, delivery points, and vehicle positions.

$L_{k}$ : position visited by the delivery vehicle $k$.

$B_{k}$ : origin of the delivery vehicle $k$.

$d_{i j k}$ : travel distance of the delivery vehicle $k$ between point $i$ and point $j,(i, j) \in A, k \in K$.

$t_{i j k}$ : travel time of the delivery vehicle between point $i$ and point $j,(i, j) \in A, k \in K$.

$s_{i k}$ : service time of the delivery vehicle $k$ at point $i, i \in(P \cup D), k \in K$.

$v_{i k}$ : cargo quantity loaded by the delivery vehicle $k$ at pickup point $i$ or unloaded at the corresponding delivery point $i, i \in(P \cup D), k \in K$.

$V_{k}$ : maximum volume limit of the delivery vehicle $k, k \in K$.

$\left(T_{a i}, T_{b i}\right)$ : service time window corresponding to point $i, i \in(P \cup D)$.

$\varepsilon_{1}$ : unit penalty cost by exceeding the earliest time window.

$\varepsilon_{2}$ : unit penalty cost by exceeding the latest time window.

$\alpha$ : unit transportation cost.

$\beta$ : unit service cost.

$\mu$ : cargo loading/unloading time factor.

(2) Decision variables

When delivery vehicle $k$ runs from point $i$ to point $j, x_{i j k}=1$. Otherwise, $x_{i j k}=0$.

$S_{i k}$ : time when the delivery vehicle $k$ starts the service from the cargo owner at point $i$, $i \in(P \cup D), k \in K$.

$V_{i k}$ : cargo capacity loaded by the delivery vehicle $k$ after serving point $i, i \in(P \cup D), k \in K$.

\subsection{Model construction}

Based on the above problem description and model hypotheses, cost minimization and maximization to the cargo owner's satisfaction were taken as the objectives. In consideration of the model-solving difficulty, the multi-objective function was transformed into a singleobjective function, that is, the concept of the time window deviation penalty cost was introduced 
to embody the index, namely, customer satisfaction. The objective function contained the transportation cost, service cost (loading/unloading time cost), and penalty cost for time window deviation, as follows:

Transportation $\operatorname{cost} C_{1}$ :

$$
C_{1}=\sum_{k \in K} \sum_{(i, j) \in A} \alpha d_{i j k} x_{i j k}
$$

Service $\operatorname{cost} C_{2}$ :

$$
C_{2}=\sum_{i \in(P \cup D)} \beta \mu v_{i k}, \forall k \in K
$$

Penalty cost for time window deviation $C_{3}$ :

$$
C_{3}=\sum_{i \in(P \cup D)} \varepsilon_{1} \max \left\{T_{a i}-S_{i k}, 0\right\}+\sum_{i \in(P \cup D)} \varepsilon_{2} \max \left\{S_{i k}-T_{b i}, 0\right\}, \forall k \in K
$$

Establishment of mathematical model:

The objective function is as follows:

$$
\min C=C_{1}+C_{2}+C_{3}
$$

The constraint condition is as follows:

$$
\begin{gathered}
\sum_{k \in K} \sum_{j \in(P \cup D)} x_{i j k}=1, \forall i \in P \\
\sum_{j \in L k} x_{i j k}-\sum_{j \in L k} x_{j, n+i, k}=0, \forall k \in K, \forall i \in P \\
\sum_{j \in(P \backslash D)} x_{B k, j, k}=1, \forall k \in K \\
S_{j k}=\mu \nu_{i k} \geq 0 \\
\sum_{i \in L k} \sum_{k \in K}\left(S_{i k}+S_{i k}+t_{i j k}\right), \forall j \in L k, i \neq j \\
S_{i k} \geq 0, \forall i \in B, \forall k \in K \\
\sum_{i, j \in(P \cup D)} \sum_{k \in K} v_{i k} x_{i j k} \leq V_{i k}, i \neq j \\
V_{i k} \geq 0, \forall i \in B, \forall k \in K
\end{gathered}
$$

Eq. (4) indicates that the objective function is the minimization of the total cost, and Eq. (5) means that each cargo owner's pickup point must and is allowed to be visited only once. According to Eq. (6), after visiting a pickup point, the vehicle must visit the corresponding delivery point, and Eq. (7) means that the vehicle starts at the corresponding origin. Eq. (8) denotes the service time at point $i$, and Eqs. (9) and (10) indicate that the time when vehicle $k$ starts the service at point $j$ is the sum of the time when it visits the previous point $i$, its service time at point $i$, and its travel time from point $i$ to point $j$, and the sum is not less than 0 . Eqs. (11) and (12) mean that vehicle $k$ meets the loading limit, and its load is greater than or equal to 0 .

\subsection{Algorithm design}

(1) Simulation of cargo transport $\mathrm{O} 2 \mathrm{O}$ platform distribution design via AnyLogic

AnyLogic is a simulation software that supports modelling by combining multiple methods. Among the methods, agent-based modelling demonstrates efficient, actual, and low-cost advantages in the simulation of vehicle scheduling and cargo distribution design for cargo transport $\mathrm{O} 2 \mathrm{O}$ platforms. Therefore, the vehicle scheduling and cargo distribution methods 
currently used by platforms were simulated via AnyLogic. In addition, based on the two methods' calculation results for the same example, the performance of the GA when used by the platforms to solve the complex VRP was verified. The vehicle scheduling and vehiclecargo matching of current cargo transport $\mathrm{O} 2 \mathrm{O}$ platforms obtained through the investigation are shown in Fig. 1.

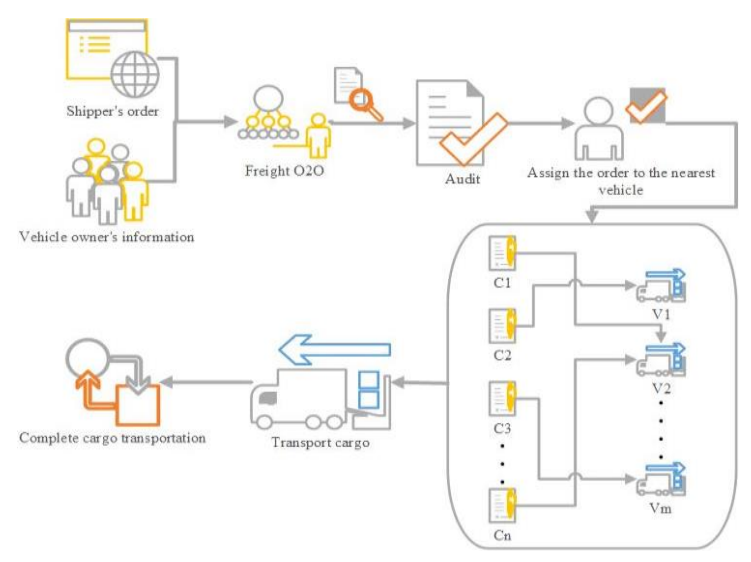

Figure 1: Vehicle-Cargo matching agents.

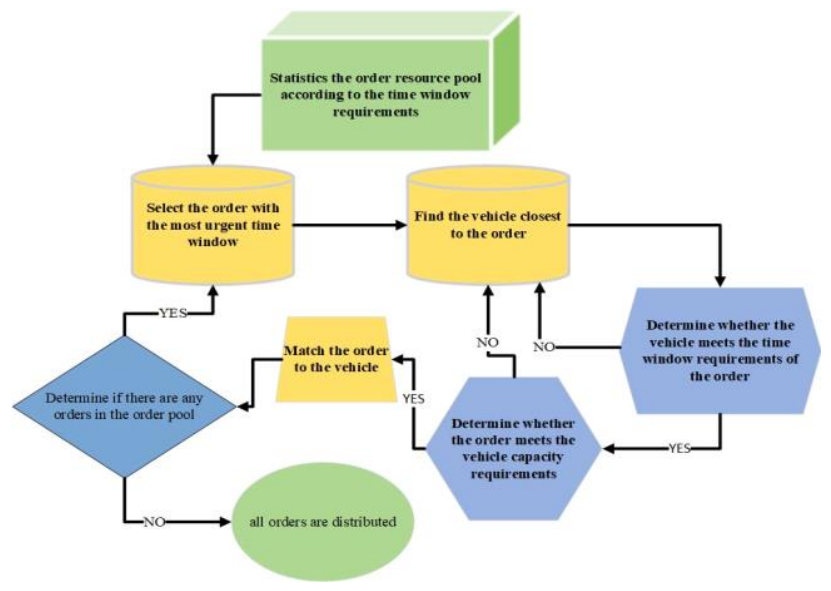

Figure 2: Cargo distribution logic of AnyLogic.

Following the aforementioned vehicle scheduling and cargo distribution process, simulation was conducted via AnyLogic, and the concrete modelling method is described below.

A new agent model was built, and the GIS graph was inserted into the main function file to simulate an actual vehicle route. The order information (e.g., vehicle, pickup point, and delivery point) obtained from the field survey was imported to the main function file using Excel sheets. Then, three new intelligent agents, namely, order, vehicle, and cargo distribution, were built in the agent model to describe the platform cargo distribution logic and process. The cargo distribution logic is shown in Fig. 2.

Step 1: Count all the cargo owner's orders, sort the orders in ascending order according to the cargo owner's pickup time window $\left(T_{a i}, T_{b i}\right)$ at the pickup point, and obtain the initial prematched order pool arranged according to the urgency priority of the delivery-taking time at the pickup point.

Step 2: Select vehicle 1 nearest to the order with the most urgent time window in the initial pre-matched order pool as the delivery vehicle. Judge whether any order for which goods must be collected exists in the order pool according to the residual volume of the vehicle. If yes, allocate the order nearest to the first pickup point to vehicle 1 (if orders with collectable goods exist at the same distance in the residual order pool, then allocate the order with the most urgent time window at the corresponding delivery point to vehicle 1). Check the residual order pool information by following this logic until vehicle 1 is loaded to its maximum volume while not being overloaded. If not overloaded, the cargo allocation to vehicle 1 is completed. After the cargo allocation is completed, the vehicle will deliver the cargo to the delivery point according to the shortest route.

Step 3: Screen the orders with the most urgent time window in the residual order pool, allocate the cargo following the logic in Step 2, and repeat the process until the cargo allocation for all the orders in the pool is completed.

(2) Solving of improved GA

The VRP with multiple constraint conditions was solved by selecting an improved GA with favourable adaptability and strong search capability. A catastrophic operation [17] was introduced to discourage the premature convergence of the GA. The solving logic of the improved GA is shown in Fig. 3. 


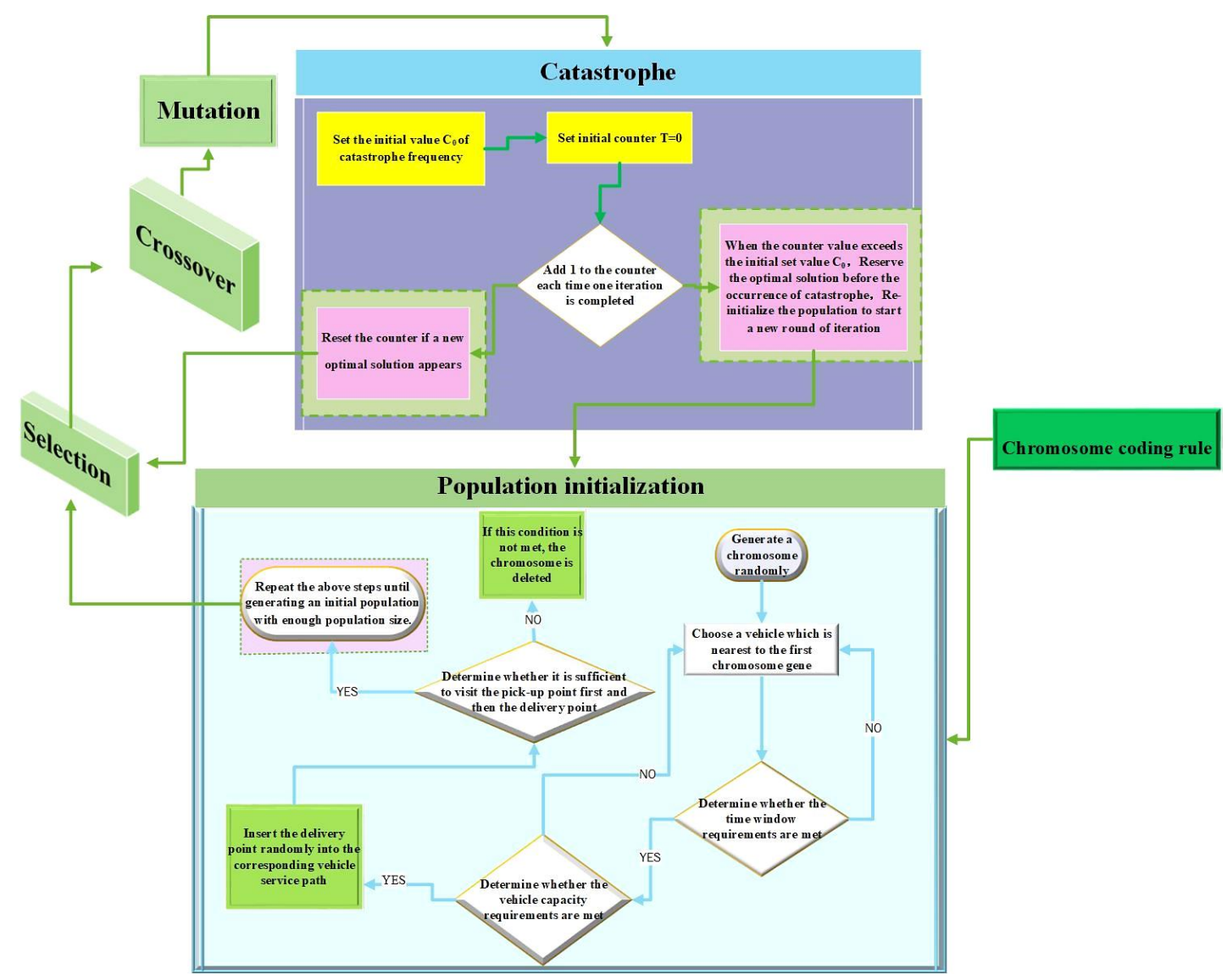

Figure 3: Solving logic of improved GA.

The concrete solving steps are as follows:

Chromosome coding rule: The pickup points of the cargo owner were denoted as $1-n$, the delivery points were denoted as $n+1-2 n$, and the vehicles were denoted as $2 n+1-2 n+m$. As the pickup point and delivery point presented a one-to-one corresponding relation in this study, first, the pickup points were randomly permutated and combined to form a chromosome during the coding process, the vehicles were inserted into multiple positions of the chromosome, and the delivery points were randomly inserted at each pickup point served by each vehicle. Taking five cargo owners and two vehicles as an example, first, a chromosome $(2,3,5,4,1)$ was randomly generated, the vehicles were inserted into the chromosome to obtain $(11,2,3,5,12$, $4,1)$, and the delivery points were inserted to acquire $(11,2,3,7,8,5,10,12,4,9,1,6)$. Finally, the formed chromosome showed that the first vehicle provided the delivery service for cargo owners 2,3 , and 5 , and the second vehicle provided the delivery service for cargo owners 1 and 4.

Population initialization: The pickup and delivery points of each cargo owner must have a one-to-one corresponding relation, that is, no. 1 pickup point corresponds to no. $n+1$ delivery point, and the delivery vehicle must visit the delivery point only after visiting the corresponding pickup point. According to the above coding rule, an infeasible solution (the delivery point is visited before the pickup point) might be generated; thus, the chromosomes that did not follow the coding rule were deleted in the generation of the initial population until an initial population with an adequate population size was generated. In addition, the nearest neighbour algorithm with a minimum cost was introduced to construct a high-quality initial solution and accelerate the convergence of the GA. The concrete steps are presented below.

Step 1: Randomly generate a chromosome with all pickup points.

Step 2: Select the vehicle nearest to the first chromosome gene, and judge the time window and volume capacity limit until the maximum vehicle loading rate is reached. 
Step 3: Randomly insert the delivery points into the corresponding vehicle service route, and judge whether the pickup point is visited before the delivery point. If not, delete this chromosome.

Step 4: Repeat the above steps until an initial population with an adequate population size is generated.

Fitness calculation: In this study, the objective function was cost minimization; thus, the objective function was transformed into a fitness function through the counting backward technique, that is, $f_{\min _{i}}=1 / F_{i}$, where $f_{\min _{i}}$ represents the fitness value of the $i^{\text {th }}$ chromosome, and $F_{i}$ is the objective function value of the $i^{\text {th }}$ chromosome.

Selection: As the cargo owner's pickup and delivery points were restricted by the vehicle visit priority order, a single-parent GA [18] was used to reduce infeasible solutions as much as possible, that is, crossover and mutation operations were conducted for each individual in the population.

Crossover: A crossover point was randomly generated in the crossover operation, and the OD pair crossover method was chosen to perform the crossover operation between the pickup points and corresponding delivery points. The crossover operation of the calculation example following the chromosome coding rule is shown in Fig. 4.

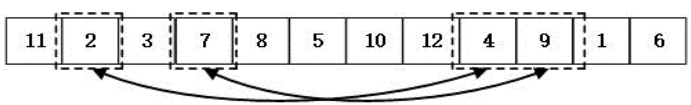

Figure 4: Chromosome crossover.

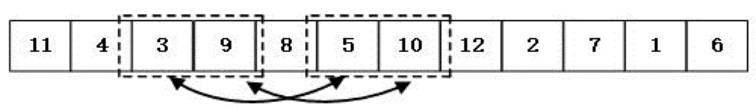

Figure 5: Chromosome mutation.

Mutation: To enhance population diversity and avoid non-feasible solutions as much as possible, a mutation position point was randomly generated, and the OD pair two-point mutation method was adopted. Meanwhile, the vehicle volume of the post-mutation chromosome was judged. If it belongs to a non-feasible solution, then the vehicles should be inserted once again according to the chromosome coding rule until a new feasible solution is generated. The post-crossover chromosome mutation process is demonstrated in Fig. 5.

Catastrophe: A catastrophic operation was introduced, that is, the local optimal solution before the catastrophe occurred was first reserved, its occurrence in nature was simulated to implement the overall "extinction" [17], and the concrete steps are as follows:

Step 1: Set the initial value $C_{0}$ of the catastrophe frequency and initial counter $T=0$.

Step 2: Add 1 to the counter each time iteration is completed. Reset the counter if a new optimal solution appears.

Step 3: When the counter value exceeds the initial set value $C_{0}$, reserve the optimal solution before the occurrence of the catastrophe, implement the catastrophic operation, then reinitialize the population to start a new round of iteration.

Termination: The maximum number of iterations was reasonably set according to the population size. The algorithm will be terminated when the number of iterations reaches the maximum value.

\section{RESULTS ANALYSIS AND DISCUSSION}

\subsection{Data acquisition}

Cargo transport O2O platforms such as Huolala, Didi Huoyun, and Yunmanbang were surveyed to determine their operating rules for cargo owners placing orders, vehicle owners receiving orders, vehicle type, and freight pricing. The related data on cargo owners' pickup and delivery points and delivery vehicles within one time period in Nanchang City, Jiangxi Province, China, as well as the model parameters were obtained through the simulation, as presented in Tables I, II, and III. 
Liu, Zhang, Wu, Meng: Vehicle Routing Problem with Soft Time Windows of Cargo ...

Table I: Information of delivery vehicles.

\begin{tabular}{|c|c|c|c|c|c|}
\hline Vehicle no. & $\begin{array}{c}\text { Longitude of vehicle } \\
\text { origin }\end{array}$ & $\begin{array}{c}\text { Latitude of } \\
\text { vehicle origin }\end{array}$ & $\begin{array}{c}\text { Vehicle } \\
\text { type }\end{array}$ & $\begin{array}{c}\text { Volume } \\
\left(\mathrm{m}^{3}\right)\end{array}$ & $\begin{array}{c}\text { Unit transportation } \\
\text { cost }(\mathrm{yuan} / \mathrm{km})\end{array}$ \\
\hline 1 & 115.894424 & 28.538767 & $\begin{array}{c}\text { Small-sized } \\
\text { minibus }\end{array}$ & 3 & 4 \\
\hline 2 & 115.941064 & 28.539465 & Iveco & 8 & 10 \\
\hline 3 & 115.937327 & 28.597011 & $\begin{array}{c}\text { Medium- } \\
\text { sized } \\
\text { minibus }\end{array}$ & 4.5 & 5 \\
\hline 4 & 115.911168 & 28.681718 & $\begin{array}{c}\text { Medium- } \\
\text { sized } \\
\text { minibus }\end{array}$ & 4.5 & 5 \\
\hline 5 & 115.970744 & 28.71714 & $\begin{array}{c}\text { Medium- } \\
\text { sized } \\
\text { minibus }\end{array}$ & 4.5 & 5 \\
\hline 6 & 115.936321 & 28.724869 & $\begin{array}{c}\text { Small-sized } \\
\text { minibus }\end{array}$ & 3 & 4 \\
\hline
\end{tabular}

Table II: Order information of cargo owners.

\begin{tabular}{|c|c|c|c|c|c|c|c|c|}
\hline No. & $\begin{array}{c}\begin{array}{c}\text { Order } \\
\text { appointment } \\
\text { time }\end{array} \\
\end{array}$ & $\begin{array}{l}\text { Longitude of } \\
\text { pickup point }\end{array}$ & $\begin{array}{c}\text { Latitude of } \\
\text { pickup } \\
\text { point }\end{array}$ & $\begin{array}{l}\text { Type of } \\
\text { goods }\end{array}$ & $\begin{array}{c}\text { Volume } \\
\left(\mathrm{m}^{3}\right)\end{array}$ & $\begin{array}{l}\text { Time window } \\
\text { requirement } \\
\text { (min) }\end{array}$ & $\begin{array}{c}\text { Longitude of } \\
\text { delivery point }\end{array}$ & $\begin{array}{c}\text { Latitude of } \\
\text { delivery point }\end{array}$ \\
\hline 1 & $5: 30$ & 115.991693 & 28.762176 & $\begin{array}{c}\text { Fast- } \\
\text { moving } \\
\text { consumer } \\
\text { goods }\end{array}$ & 1.4 & \pm 10 & 115.866392 & 28.643984 \\
\hline 2 & $7: 30$ & 115.88235 & 28.788643 & Apparatus & 4 & \pm 20 & 115.845192 & 28.669024 \\
\hline 3 & $9: 30$ & 115.849508 & 28.754196 & $\begin{array}{c}\text { Fast- } \\
\text { moving } \\
\text { consumer } \\
\text { goods }\end{array}$ & 1.2 & \pm 5 & 115.939554 & 28.500808 \\
\hline 4 & $8: 30$ & 115.829508 & 28.684196 & Apparatus & 2 & \pm 8 & 115.928771 & 28.707175 \\
\hline 5 & $5: 30$ & 115.879508 & 28.654196 & Food & 0.8 & \pm 15 & 116.009331 & 28.706732 \\
\hline 6 & $6: 30$ & 115.801934 & 28.580709 & $\begin{array}{c}\text { Fast- } \\
\text { moving } \\
\text { consumer } \\
\text { goods }\end{array}$ & 3 & \pm 15 & 115.878609 & 28.754116 \\
\hline 7 & $5: 30$ & 116.008832 & 28.540734 & Apparatus & 1 & \pm 10 & 115.876669 & 28.719593 \\
\hline 8 & $7: 30$ & 115.911308 & 28.554298 & \begin{tabular}{|c|} 
Office \\
supplies
\end{tabular} & 0.5 & \pm 13 & 115.804377 & 28.738045 \\
\hline 9 & $10: 30$ & 115.876238 & 28.547952 & $\begin{array}{c}\text { Office } \\
\text { supplies }\end{array}$ & 0.8 & \pm 15 & 115.876673 & 28.700286 \\
\hline 10 & 5:00 & 115.984825 & 28.626484 & Apparatus & 5 & \pm 10 & 115.889681 & 28.68045 \\
\hline
\end{tabular}

Table III: Model parameters.

\begin{tabular}{|c|l|c|}
\hline Parameter & \multicolumn{1}{|c|}{ Definition } & Value \\
\hline$\beta$ & Unit loading/unloading time cost (yuan/hour) & 60 \\
\hline$\varepsilon_{1}$ & Unit penalty cost if earlier than the earliest time window (yuan/hour) & 50 \\
\hline$\varepsilon_{2}$ & Unit penalty cost if later than the latest time window (yuan/hour) & 70 \\
\hline$\mu$ & Cargo loading/unloading time factor (hour $/ \mathrm{m}^{3}$ ) & 0.1 \\
\hline$\delta$ & Average vehicle running speed $(\mathrm{km} / \mathrm{hour})$ & 30 \\
\hline
\end{tabular}




\subsection{Results analysis of calculation example}

The GA was coded using C\# programming language, and the initial population size, maximum number of iterations, crossover probability, and mutation probability were set to $200,500,0.85$, and 0.04 , respectively.

For solving with the improved GA, the distance between two points on a spherical surface was calculated using the haversine formula to calculate the distance between every two vehicles and pickup and delivery points, whereas the AnyLogic simulation directly calculated the distance of the built-in GIS road networks. To compare the operation results accurately, the haversine formula was used uniformly to correct the optimal scheme result obtained by AnyLogic. The scheme results obtained by the two methods are listed in Table IV.

Table IV: Algorithm results.

\begin{tabular}{|c|c|c|c|c|c|}
\hline Scheme & $\begin{array}{c}\text { Vehicle-cargo matching } \\
\text { route }\end{array}$ & $\begin{array}{c}\text { Transportation } \\
\text { cost (yuan) }\end{array}$ & $\begin{array}{c}\text { Service cost } \\
\text { (yuan) }\end{array}$ & $\begin{array}{c}\text { Time window } \\
\text { deviation cost (yuan) }\end{array}$ & $\begin{array}{c}\text { Total cost } \\
\text { (yuan) }\end{array}$ \\
\hline $\begin{array}{l}\text { AnyLogic } \\
\text { simulation }\end{array}$ & $\begin{array}{l}\text { Vehicle } 1-7-9-19-17 \\
\text { Vehicle 2-10-5-4-15-14-20 } \\
\text { Vehicle 3-6-8-16-18 } \\
\text { Vehicle 4-2-12 } \\
\text { Vehicle 6--1-3-11-13 }\end{array}$ & 1379.14 & 236.4 & 511.17 & 2126.71 \\
\hline $\begin{array}{l}\text { Improved } \\
\text { GA }\end{array}$ & $\begin{array}{l}\text { Vehicle 1-8-18-3-13 } \\
\text { Vehicle 2-7-17-2-12 } \\
\text { Vehicle 3-10-20-5-15 } \\
\text { Vehicle 4-1-11-6-16 } \\
\text { Vehicle 5-4-14 } \\
\text { Vehicle 6-9-19 }\end{array}$ & 1459.707 & 236.4 & 59.402 & 1755.51 \\
\hline
\end{tabular}

Note: The pickup points are numbered 1-10, and the corresponding delivery points are numbered 11-20.

The results obtained by the two algorithms were compared, as shown in Fig. 6.

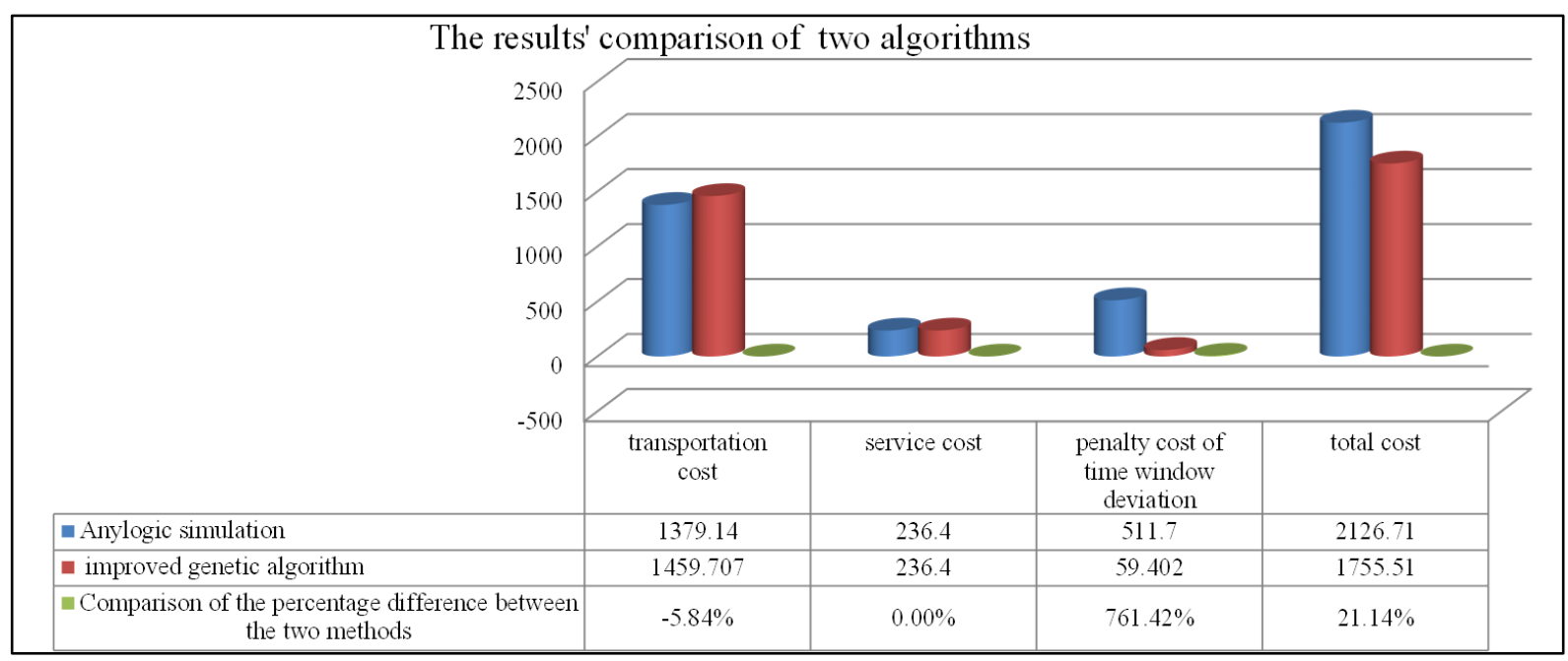

Figure 6: Comparison of algorithm results.

The algorithm results showed that the transport cost obtained by the improved GA was $5.84 \%$ higher than that obtained by the cargo transport platform nearest matching scheme simulated by AnyLogic. However, the time window deviation cost obtained by the cargo transport platform nearest matching scheme simulated by AnyLogic was $761.42 \%$ higher than that obtained by the improved GA. Finally, the total cost obtained by the improved GA was $21.14 \%$ lower than that obtained by the cargo transport platform nearest matching scheme simulated by AnyLogic. 


\section{CONCLUSION}

To minimize the comprehensive cost of vehicle and cargo owners, the vehicle scheduling and vehicle-cargo matching problems of cargo transport $\mathrm{O} 2 \mathrm{O}$ platforms were explored in this study, and conditions closest to reality were taken as the constraint conditions. Moreover, the optimal allocation of vehicle and cargo owners' order resources on cargo transport $\mathrm{O} 2 \mathrm{O}$ platforms was realized, aiming for a global optimal solution. The nearest matching scheme results simulated by AnyLogic and improved GA were analysed comparatively, and the following conclusions were drawn:

(1) The time window deviation cost obtained by the nearest matching scheme simulated by AnyLogic was much higher than that obtained by the improved GA, which accords with actual situations. Presently, cargo owners placing orders through platforms have high requirements for timeliness; thus, a decline in timeliness can easily lead to reduced customer satisfaction.

(2) The total cost obtained by the improved GA was lower than that obtained by the commonly used nearest matching scheme based on the AnyLogic simulation. The main reason behind this result was that the time window deviation cost was too high during the nearest matching.

(3) The overall performance of the improved GA was better than that of the nearest matching scheme, which is commonly used in cargo transport $\mathrm{O} 2 \mathrm{O}$ platforms. The improved GA aimed to obtain an economical and efficient scheme from the global optimum perspective, whereas the primary goal of the nearest matching scheme based on the AnyLogic simulation was the shortest route. Thus, the introduction of efficient and economical algorithms, which can maximize vehicle owners' revenues and cargo owners' satisfaction, is an inevitable choice for cargo transport $\mathrm{O} 2 \mathrm{O}$ platforms.

Although ordered cargoes were classified in this study, only the influence of the cargo volume on the loading/unloading time and total cost of the vehicle owner was considered. However, in actual situations, different types of cargoes may not be combined owing to their different natures. Thus, platforms should first classify cargoes according to the cargo information. When optimizing the vehicle-cargo matching scheme, platforms should first judge whether the different cargoes loaded on the same vehicle can be combined, which will be the topic of a follow-up study. In addition, the performance of hyper-heuristic algorithms, such as the improved GA, ant colony algorithm, and quantum adiabatic evolution algorithm, in solving the vehicle-cargo matching and vehicle routing optimization scheme of cargo transport $\mathrm{O} 2 \mathrm{O}$ platforms should be compared, which is another follow-up study direction expected to provide platforms with efficient, high-quality, and economical algorithm schemes.

\section{ACKNOWLEDGEMENT}

The study was supported by the Humanities and Social Sciences Research Funding Project of Universities and Colleges in Jiangxi (No. GL20223) (No. GL18247) and National Natural Science Foundation of China (No. 71940009).

\section{REFERENCES}

[1] Dantzig, G. B.; Ramser, J. H. (1959). The truck dispatching problem, Management Science, Vol. 6, No. 1, 80-91, doi:10.1287/mnsc.6.1.80

[2] Taillard, É.; Badeau, P.; Gendreau, M.; Guertin, F.; Potvin, J.-Y. (1997). A tabu search heuristic for the vehicle routing problem with soft time windows, Transportation Science, Vol. 31, No. 2, 170-186, doi: $10.1287 / \operatorname{trsc} .31 .2 .170$

[3] Baldacci, R.; Bartolini, E.; Mingozzi, A. (2011). An exact algorithm for the pickup and delivery problem with time windows, Operations Research, Vol. 59, No. 2, 414-426, doi:10.1287/ opre. 1100.0881 
[4] Li, J.; Zheng, Y.; Dai, B.; Yu, J. (2020). Implications of matching and pricing strategies for multiple-delivery-points service in a freight $\mathrm{O} 2 \mathrm{O}$ platform, Transportation Research Part E: Logistics and Transportation Review, Vol. 136, Paper 101871, 15 pages, doi:10.1016/ j.tre.2020.101871

[5] Lagos, C.; Guerrero, G.; Cabrera, E.; Moltedo, A.; Johnson, F.; Paredes, F. (2018). An improved particle swarm optimization algorithm for the VRP with simultaneous pickup and delivery and time windows, IEEE Latin America Transactions, Vol. 16, No. 6, 1732-1740, doi:10.1109/TLA.2018.8444393

[6] Erdoan, G. (2017). An open source spreadsheet solver for vehicle routing problems, Computers \& Operations Research, Vol. 84, 62-72, doi:10.1016/j.cor.2017.02.022

[7] Montero, A.; Miranda-Bront, J. J.; Méndez-Díaz, I. (2017). An ILP-based local search procedure for the VRP with pickups and deliveries, Annals of Operations Research, Vol. 259, No. 1, 327 350, doi:10.1007/S10479-017-2520-5

[8] Tiwari, S.; Kumar, A.; Punhani, A.; Mishra, K. K. (2019). Modified environmental adaptation method and its application in test case generation, Journal of Engineering Science and Technology Review, Vol. 12, No. 6, 208-218, doi:10.25103/jestr.126.26

[9] Alaia, E. B.; Harbaoui, I.; Borne, P.; Bouchriha, H. (2018). A comparative study of the PSO and GA for the m-MDPDPTW, International Journal of Computers Communications \& Control, Vol. 13, No. 1, 8-23, doi:10.15837/IJCCC.2018.1.2970

[10] Jiang, H. (2020). Solving multi-robot picking problem in warehouses: a simulation approach, International Journal of Simulation Modelling, Vol. 19, No. 4, 701-712, doi:10.2507/IJSIMM19$\underline{4-\mathrm{CO} 19}$

[11] Bulhões, T.; Hà, M. H.; Martinelli, R.; Vidal, T. (2018). The vehicle routing problem with service level constraints, European Journal of Operational Research, Vol. 265, No. 2, 544-558, doi:10.1016/J.EJOR.2017.08.027

[12] Gallegos Acosta, A. E.; Torres Soto, M. D.; Alvarez Rodriguez, F. J.; Torres Soto, A. (2020). Manipulation of metaheuristics for the search of typical testers, Dyna-Ingenieria e Industria, Vol. 95, No. 1, 11 pages, doi: $10.6036 / 9375$

[13] Campelo, P.; Neves-Moreira, F.; Amorim, P.; Almada-Lobo, B. (2019). Consistent vehicle routing problem with service level agreements: A case study in the pharmaceutical distribution sector, European Journal of Operational Research, Vol. 273, No. 1, 131-145, doi:10.1016/ J.EJOR.2018.07.030

[14] Şevkli, A. Z.; Güler, B. (2017). A multi-phase oscillated variable neighbourhood search algorithm for a real-world open vehicle routing problem, Applied Soft Computing, Vol. 58, 128-144, doi:10.1016/J.ASOC.2017.04.045

[15] Hannan, M. A.; Akhtar, M.; Begum, R. A.; Basri, H.; Hussain, A.; Scavino, E. (2018). Capacitated vehicle-routing problem model for scheduled solid waste collection and route optimization using PSO algorithm, Waste Management, Vol. 71, 31-41, doi:10.1016/j.wasman.2017.10.019

[16] Poonthalir, G.; Nadarajan, R. (2018). A fuel efficient green vehicle routing problem with varying speed constraint (F-GVRP), Expert Systems with Applications, Vol. 100, 131-144, doi:10.1016/J.ESWA.2018.01.052

[17] Liao, M.; Zhang, Y. (2005). Study on the effect of cataclysm operator on genetic algorithm, Computer Engineering and Applications, Vol. 41, No. 13, 54-56

[18] Ünal, A. N.; Kayakutlu, G. (2016). A partheno-genetic algorithm for dynamic 0-1 multidimensional Knapsack problem, RAIRO - Operations Research, Vol. 50, No. 1, 47-66, doi:10.1051/RO/2015011 